\title{
Impulsive State Feedback Control of the Rhizosphere Microbial Degradation in the Wetland Plant
}

\author{
Zhong Zhao, Liuyong Pang, Zhanping Zhao, and Chengguang Luo \\ Department of Mathematics, Huanghuai University, Zhumadian, Henan 463000, China \\ Correspondence should be addressed to Zhong Zhao; zhaozhong8899@163.com
}

Received 13 October 2014; Revised 23 February 2015; Accepted 23 February 2015

Academic Editor: Carlo Piccardi

Copyright (c) 2015 Zhong Zhao et al. This is an open access article distributed under the Creative Commons Attribution License, which permits unrestricted use, distribution, and reproduction in any medium, provided the original work is properly cited.

\begin{abstract}
The rhizosphere microbe plays an important role in removing the pollutant generated from industrial and agricultural production. To investigate the dynamics of the microbial degradation, a nonlinear mathematical model of the rhizosphere microbial degradation is proposed based on impulsive state feedback control. The sufficient conditions for existence of the positive order-1 or order- 2 periodic solution are obtained by using the geometrical theory of the semicontinuous dynamical system. We show the impulsive control system tends to an order-1 periodic solution or order- 2 periodic solution if the control measures are achieved during the process of the microbial degradation. Furthermore, mathematical results are justified by some numerical simulations.
\end{abstract}

\section{Introduction}

The constructed wetland is regarded as the most efficient and cost-effective system that involves microbes consuming pollutant generated from industrial and agricultural production. However, degradation ability of the microbe in the constructed wetland is affected by many factors such as temperature, $\mathrm{pH}$, dissolved oxygen, and substrate concentration [1]. Studies have shown that excessive pollutant strongly affects the dynamic behavior of microbe, which can lead to the incidence of the sustained oscillation and decrease degradation ability of the microbe under certain conditions [2]. For the purpose of improving degradation ability of the microbe and decreasing the inhibition effect of the substrate, it is necessary to control the pollutant concentration lower than a certain level.

The removal of the sewage from a water body by the biodegradation has been investigated by many researchers [3-15]. In [14], pollutant removal process of a constructed wetland is simulated by the following model:

$$
\begin{aligned}
\frac{d T}{d t} & =f(S) T-\sigma T, \\
\frac{d W}{d t} & =Q-(\gamma+\alpha) W,
\end{aligned}
$$

$$
\frac{d S}{d t}=\gamma W-\frac{f(S) T}{Y}-\phi S,
$$

where $f(S)$ denotes the mangrove growth rate which is chosen to follow Monod kinetics $(f(S)=\beta S /(k+S), \beta>$ $0, k>0) . T(t)$ is the mangrove biomass concentration, $W(t)$ denotes the total nitrogen concentration in wastewater, and $S(t)$ shows the total nitrogen concentration in soil solution. The meaning of other parameters is also given in [14]. Scott et al. [15] propose a mathematical model for dispersal of bacterial inoculants colonizing the water rhizosphere to describe bacterial growth and movement in the rhizosphere.

Recently, there are many studies on the geometric theory of semicontinuous dynamical system which are applied into the chemostat model [16-20]. Considering the product inhibition during the microorganism culture, Guo and Chen [16] develop a mathematical model concerning a chemostat with impulsive state feedback control to investigate the periodicity of bioprocess and an order-1 periodic solution is obtained by the existence criteria of periodic solution. The mathematical model of a continuous bioprocess with impulsive state feedback control is formulated [18] and the analysis of the bioprocess stability is presented. As far as I know, little information is available concerning the dynamical research on the microbial degradation of the rhizosphere. 
The main purpose of this paper is to construct a mathematical model of the microbial degradation with impulsive state feedback control and understand the dynamics of the microbial degradation in the constructed wetland.

The paper is organized as follows: a mathematical model of the microbial degradation with impulsive state feedback control is proposed in Section 2. In Section 3, the qualitative analysis of system without impulsive control is given. Furthermore, the existence of order- 1 and order- 2 periodic solutions is investigated in Section 4. Finally, we give some numerical simulations and a brief discussion.

\section{Model Description and Preliminaries}

In the constructed wetland, it is showed that the rhizosphere microbe makes an important contribution to the degradation of pollutant $[1,21]$. Based on references $[1,16-21]$, we give some assumptions to investigate the dynamics of the microbial degradation of the rhizosphere.

(a) Suppose the pollutant and rhizosphere microbe are uniformly distributed inside the rhizosphere. $S(t)$ denotes the pollutant concentration discharged from the household and industrial sources. $x(t)$ is the concentration of the rhizosphere microbe.

(b) It is assumed the pollutant is discharged into the plant rhizosphere from outside with a constant $Q$.

(c) $\alpha$ is the rate of decrease due to biochemical and other factors, which is proportional to the pollutant concentration $S(t)$.

(d) The growth of the rhizosphere microbe is assumed to follow the Monod equation involving the pollutant concentration $S(t)$ as well as the microbial concentration $x(t)$ (i.e., $\mu S x / \delta(K+S)$ ), where $\mu$ is the maximum specific growth rate and the constant $\delta$ is yield term and $K$ is a half-saturation constant.

(e) The mortality of the rhizosphere microbe is denoted as $D$. Considering the nutrient recycling, we suppose the fraction of the death microbe is converted into the substrate and $r(0<r \leq 1)$ is the fraction of the conversion.

(f) Let $h$ show a predetermined threshold, which is a minimum allowed value harmless to human health. When pollutant concentration is higher than the critical threshold $h$ (which can be measured in advance), control measures are taken to reduce the pollutant concentration below the critical threshold; that is, we will inoculate the microbe into the plant rhizosphere so as to improve microbial ability of degradation and $\tau$ denotes the amount of releasing the microbe into the plant rhizosphere. At the same time, we will control the emissions of pollutant. $E(0<E<1)$ is the effect of controlling the pollutant sources.
According to the above assumption, we formulate the following model:

$$
\begin{aligned}
& \frac{d S}{d t}=Q-\alpha S-\frac{\mu S x}{\delta(K+S)}+r D x, \\
& \frac{d x}{d t}=\frac{\mu S x}{(K+S)}-D x, \\
& \qquad S<h, \\
& \Delta S=-E S, \\
& \Delta x=\tau,
\end{aligned}
$$

$$
S=h,
$$

where $\Delta S=S\left(t^{+}\right)-s(t)$ and $\Delta x=x\left(t^{+}\right)-x(t)$.

Next, we will discuss the existence of periodic solution of (2) by using the geometry theory of semicontinuous dynamic system. For convenience, we give some definitions and lemmas.

Definition 1 (see [22]). Let $M$ denote the impulsive set and $N$ be the phase set. Suppose $g: N \rightarrow N$ is a mapping. For any point $P \in N$, there exists $t_{1}>0$ such that $F(P)=f\left(P, t_{1}\right)=$ $P_{1} \in M, P_{1}^{+}=\varphi\left(P_{1}\right) \in N$, and then $g(P)=l\left(P_{1}^{+}\right)-l(P)$ is called the successor function of point $P$ and the point $P_{1}^{+}$is called the successor point of $P$.

To investigate the dynamics of system (2), we construct the following Pioncaré map. Choose two sections $P_{0}$ and $P_{1}$ as the Poincaré sections, where $P_{0}=\{(S, x) \mid S=(1-$ $E) h, x \geq \tau\}$ and $P_{1}=\{(S, x) \mid S=h, x \geq 0\}$. Assume the point $B_{k}\left(h, x_{k}\right)$ is on the Pioncaré section $P_{1}$, then $B_{k}^{+}((1-$ $\left.E) h, x_{k+1}\right)$ lies on the section $P_{0}$ due to the impulsive effect, and the trajectory starting from the initial value $B_{k}^{+}$intersects the section $P_{1}$ at the point $B_{k+1}=\left(h, x_{k+1}\right)$, where $x_{k+1}$ is determined by $x_{k}$ and the parameter $\tau$. Therefore, we get the Pioncaré map as follows:

$$
x_{k+1}=F\left(\tau, x_{k}\right) \text {. }
$$

\section{Qualitative Analysis for System (2)}

Before discussing the periodic solution of system (2), we should consider the qualitative property of (2) without the impulsive effect.

Lemma 2. Suppose $\omega(t)=(S(t), x(t))$ is a solution of (2) subject to $\omega\left(0^{+}\right) \geq 0$, then $\omega(t) \geq 0$ for all $t \geq 0$, and furthermore $\omega(t)>0, t \geq 0$ if $\omega\left(0^{+}\right)>0$.

\section{Theorem 3. The system (2) is ultimately bounded.}

Proof. Define a function $V(t)=S(t)+x(t) / \delta$. We calculate the upper right derivative of $V(t)$ along a solution of the first and second equations of system (2) and get the following differential equation:

$$
\frac{d V}{d t}=Q-\alpha S+\left(r-\frac{1}{\delta}\right) D x \leq Q-\beta V(t),
$$


where $\beta=\min \{\alpha,(1 / \delta-r) D \delta\}$. We have

$$
S(t)+\frac{x(t)}{\delta} \leq \lim _{t \rightarrow \infty} V(t)=\frac{Q}{\beta} .
$$

According to the positivity of $S(t)$ and $x(t)$, we obtain $S(t) \leq$ $Q / \beta$ and $x(t) \leq Q / \beta$. The proof is completed.

Next, we explore the asymptotical behavior of the system (2) without impulsive effect.

$$
\begin{aligned}
& \frac{d S}{d t}=Q-\alpha S-\frac{\mu S x}{\delta_{1}(K+S)}+r D x, \\
& \frac{d x}{d t}=\frac{\mu S x}{(K+S)}-D x .
\end{aligned}
$$

An equilibrium point of system (6) satisfies the system

$$
\begin{array}{r}
Q-\alpha S-\frac{\mu S x}{\delta_{1}(K+S)}+r D x=0, \\
\frac{\mu S x}{(K+S)}-D x=0 .
\end{array}
$$

It can be seen that system (7) has a microbe-free equilibrium of the form $E_{0}=(Q / \alpha, 0)$. We start by analyzing the behavior of the system (7) near $E_{0}$. The characteristic equation of the linearization of (6) near $E_{0}$ is

$$
\operatorname{det}\left(\begin{array}{cc}
-\alpha-\lambda & r D \\
0 & \frac{\mu Q}{k \alpha+Q}-D-\lambda
\end{array}\right)=0 .
$$

Two eigenvalues are $\lambda_{1}=-\alpha$ and $\lambda_{2}=\mu \mathrm{Q} /(k \alpha+\mathrm{Q})-$ $D$, respectively. We obtain the microbe-free equilibrium of system (6) is unique and locally asymptotically stable if the condition $\mu \mathrm{Q} /(k \alpha+Q)<D$ holds.

Define $R_{1}=\mu Q / D(k \alpha+Q)$.

Theorem 4. The microbe-free equilibrium $E_{0}$ is asymptotically stable if $R_{1}<1 . E_{0}$ is unstable if $R_{1}>1$.

From (7), it follows that when the trivial equilibrium $E_{0}$ of system (6) is asymptotically stable then positive equilibrium does not exist. When $R_{1}>1$, system (6) has a unique positive equilibrium $E_{1}\left(S^{*}, x^{*}\right)$, where $S^{*}=D k /(\mu-D)$, $x^{*}=\delta\left(Q-\alpha S^{*}\right)\left(k+S^{*}\right) /\left((\mu-r D \delta) S^{*}-r D \delta k\right)=\delta(Q \mu-Q D-$ $\alpha D k) / \mu D k(\mu-D)(1-r \delta), \mu>D$. We can easily obtain $S^{*}>0$ and $x^{*}>0$ for $R_{1}>1$. Next, we analyze the stability of the positive equilibrium $E^{*}\left(S^{*}, x^{*}\right)$. The characteristic equation at $E^{*}\left(S^{*}, P^{*}\right)$ is

$$
\operatorname{det}\left(\begin{array}{cc}
\lambda+\alpha+\frac{\mu k x^{*}}{\delta\left(k+S^{*}\right)^{2}} & -r D+\frac{\mu S^{*}}{\delta\left(k+S^{*}\right)} \\
-\frac{\mu k x^{*}}{\left(k+S^{*}\right)^{2}} & \lambda+D-\frac{\mu S^{*}}{k+S^{*}}
\end{array}\right)=0 .
$$

We know $\lambda^{2}+\left(\alpha+\mu k x^{*} / \delta\left(k+S^{*}\right)^{2}\right) \lambda+\left(\mu k x^{*} /(k+\right.$ $\left.\left.S^{*}\right)^{2}\right)\left(\mu S^{*} / \delta\left(k+S^{*}\right)-r D\right)=0$. After a few computations, we have $\lambda_{1}+\lambda_{2}<0, \lambda_{1} \lambda_{2}>0$ for $R_{1}>1$. Therefore, we have.
Theorem 5. If $R_{1}>1$ holds, the positive equilibrium of system (6) is locally asymptotically stable.

Now, let us discuss the global stability of system (2). Firstly, we will give the following lemma.

Lemma 6. Suppose $\Gamma(t)=(S(t), x(t))$ is a periodic orbit of the system (2) with the period $T$. The bounded region $R$ consists of all the points in phase plane $\Gamma$. Denote

$$
\Delta=\int_{0}^{T}\left[\frac{\partial f_{1}}{\partial S}(S(t), x(t))+\frac{\partial f_{2}}{\partial x}(S(t), x(t))\right] d t,
$$

where $d S / d t \triangleq f_{1}(S, x)$ and $d x / d t \triangleq f_{2}(S, x)$. Then we obtain

$$
\begin{aligned}
\Delta= & -\int_{0}^{T}\left[\alpha+\frac{\mu k x(t)}{\delta_{1}(k+S(t))^{2}}\right] d t \\
& +\int_{0}^{T}\left[\frac{\mu S(t)}{k+S(t)}-D\right] d t \leq 0 .
\end{aligned}
$$

Proof. From system (2), we obtain $\int_{0}^{T}[(\mu S(t) /(k+S(t))-$ $D] d t=\int_{0}^{T} d(\ln x(t))$. Obviously, we have $\int_{0}^{T}(1 / x)(d x / d t)=$ 0 because of the periodicity of $x(t)$. Therefore, we obtain $\Delta=-\int_{0}^{T}\left[\alpha+\mu k x(t) / \delta_{1}(k+S(t))^{2}\right] d t \leq 0$.

Theorem 7. The positive equilibrium $E^{*}\left(S^{*}, x^{*}\right)$ is globally asymptotically stable if $R_{1}>1$, where $R_{1}$ is defined above.

Proof. From Theorem 5, we know that $E^{*}\left(S^{*}, x^{*}\right)$ is locally asymptotically stable. According to Lemma 6, we obtain the periodic solution is stable if it exists around $E^{*}\left(S^{*}, x^{*}\right)$. However, it is impossible according to Poincare-Bendixson Theorem, which implies that $E^{*}\left(S^{*}, x^{*}\right)$ is globally asymptotically stable.

\section{Existence of the Periodic Solution}

When $h<k D /(\mu-D)$, the trajectory from the initial value $(S(0), x(0))$ with $S(0)<h$ will intersect the Poincare section $P_{1}$ infinite times; while $h \geq k D /(\mu-D)$, the trajectory from the initial value $(S(0), x(0))$ with $S(0)<h$ will tend to the equilibrium $\left(S^{*}, x^{*}\right)$ after the impulsive effect of the finite times. In order to investigate the impact of the impulsive effect on the system (2), we have two cases: (a) $h<k D /(\mu-D)$ and (b) $h \geq k D /(\mu-D)$.

4.1. Existence of the Order-1 Periodic Solution for $h<k D /(\mu-$ $D)$. The impulsive set $S=h$ intersects the isoclinal line $d S / d t=0$ at the point $D\left(h, x_{D}\right)$, where $x_{D}$ is satisfied $x_{D}=$ $\delta(Q-\alpha h)(k+h) /((\mu-r D \delta) h-r D \delta k)$. The phase set $S=$ $(1-E) h$ intersects the isoclinal line $d S / d t=0$ at the point $H\left((1-E) h, x_{H}\right)$, where $x_{H}$ is satisfied $x_{H}=\delta(Q-\alpha(1-$ $E) h)(k+(1-E) h) /((\mu-r D \delta)(1-E) h-r D \delta k)$.

Choose $A_{1}((1-E) h, \varepsilon)$, where $\varepsilon$ is small enough and $\varepsilon<\tau$. According to the qualitative analysis, the trajectory from the point $A_{1}$ reaches the point $B_{1}\left(h, \varepsilon_{1}\right)$ on the Poincare section $P_{1}$, next jumps onto the point $A_{2}\left((1-E) h, \varepsilon_{1}+\tau\right)$, and then 


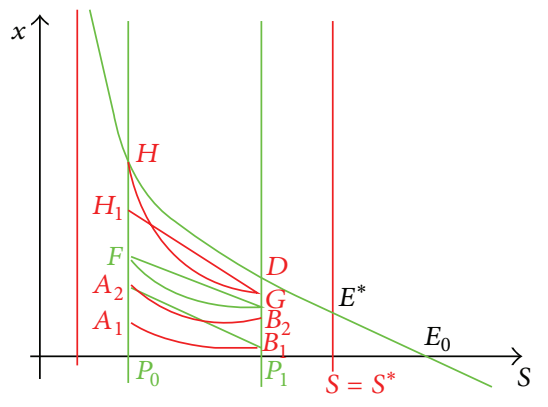

(a)

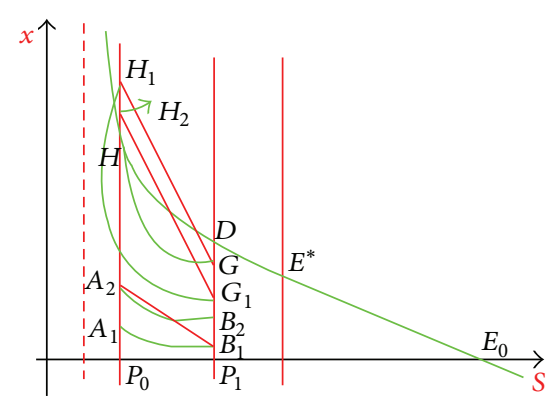

(b)

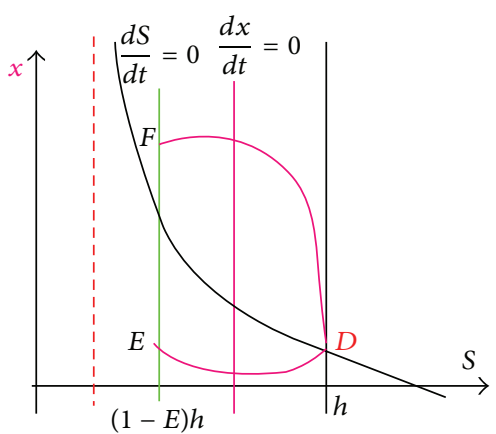

(c)

FIgure 1: (a) and (b) The existence of order-1 periodic solution for $h<k D /(\mu-D)$. (c) The existence of order-1 or order-2 periodic solution for $h \geq k D /(\mu-D)$.

reaches the point $B_{2}\left(h, \varepsilon_{2}\right)$ again. Obviously, the point $A_{2}$ is above the point $A_{1}$ due to impulsive effect $\Delta x=\tau$ and $\tau+\varepsilon_{1}>$ $\varepsilon$. Therefore, The point $A_{2}$ is the successor point of the point $A_{1}$, and in view of the property of the successor function, we have

$$
f\left(x_{A_{2}}\right)-f\left(x_{A_{1}}\right)>0 .
$$

Suppose the trajectory from the point $H\left((1-E) h, x_{H}\right)$ interacts the Poincaré section $P_{1}$ at the point $G(h, l)$ and next jumps onto the Poincaré section $P_{0}$ at the point $H_{1}((1-$ $\left.E) h, \gamma_{1}\right)$. There are two possible cases between the point $H$ and $H_{1}$. One is that the point $H_{1}$ is below the point $H$, and the other case is that point $H_{1}$ is above the point $H$.

For the point $H_{1}$ being below the point $H$ (see Figure 1(a)), the point $H_{1}$ is the successor point of the point $H$, we have

$$
f\left(x_{H_{1}}\right)-f\left(x_{H}\right)<0 .
$$

Hence, there must be a point $F\left((1-E) h, x_{F}\right)$ such that $f\left(x_{F}\right)=$ 0 ; that is, system (2) has an order-1 periodic solution.

When the point $H_{1}$ is above the point $H$, the trajectory from the point $H_{1}$ interacts the Poincaré section at the point $G_{1}\left(h, l_{1}\right)$ and next jumps onto the point $H_{2}\left((1-E) h, \gamma_{2}\right)$. Obviously, the point $H_{2}$ is below the point $H_{1}$ according to the impulsive effect and qualitative property of system (2) (see Figure 1(b)). The point $\mathrm{H}_{2}$ is the successor point of the point $H_{1}$; we obtain

$$
f\left(x_{H_{2}}\right)-f\left(x_{H_{1}}\right)<0 .
$$

From (12) and (14), we obtain system (2) exists an order-1 periodic solution.

4.2. Existence of the Periodic Solution for $h \geq k D /(\mu-D)$. Suppose the Poincaré section $S=h$ interacts the isoclinal line $d S / d t=0$ at the point $D\left(h, x_{D}\right)$ and the Poincare section $S=(1-E) h$ intersects the isoclinal line $d S / d t=0$ at the point $H\left((1-E) h, x_{H}\right)$. There must exist a trajectory $\Gamma$ passing through the point $D$ and tangent to the Poincaré section $S=h$ at the point $D\left(h, x_{D}\right)$ with $x_{D}=\delta(Q-\alpha h)(k+$ $h) /((m u-r D \delta) h-r D \delta k$ ) (see Figure 1(c)). Furthermore, the trajectory $\Gamma$ crosses the Poincaré section $S=(1-E) h$ at the point $E\left((1-E) h, \bar{x}_{1}\right)$ and the point $F\left((1-E) h, \bar{x}_{2}\right)$ with $\bar{x}_{1}<\delta(Q-\alpha(1-E) h)(k+(1-E) h) /((m u-r D \delta)(1-$ $E) h-r D \delta k)<\bar{x}_{2}$. For $x \in\left(\bar{x}_{1}, \bar{x}_{2}\right)$, the trajectory passing through the point $((1-E) h, x)$ will not interact with the Poincaré section $S=h$ and will ultimately tend to the positive equilibrium $E^{*}\left(S^{*}, x^{*}\right)$. For any trajectory of (2) will pass through the point $((1-E) h, x), x \in\left(\bar{x}_{1}, \bar{x}_{2}\right)$ after some impulsive effects on the Poincaré section $P_{1}$, they ultimately tend to the positive equilibrium $E^{*}\left(S^{*}, x^{*}\right)$ and there is not any periodic solution. For $\tau>\bar{x}_{2}$, a trajectory of system (2) will intersect the Pioncaré section $S=(1-E) h$ infinite times due to the impulsive effect $\Delta S=-E S, \Delta x=\tau$. When the condition $\tau>\bar{x}_{2}$ is satisfied, we have $0<B_{i}\left(h, x_{i}\right)<$ $B_{j}\left(h, x_{j}\right)<\delta(Q-\alpha h)(k+h) /((m u-r D \delta) h-r D \delta k)$ for any two points $B_{i}\left(h, x_{i}\right)$ and $B_{j}\left(h, x_{j}\right)$. The corresponding impulsive points $B_{i}^{+}\left(h, x_{i}+\tau\right)$ and $B_{j}^{+}\left(h, x_{j}+\tau\right)$ are above the point $F$. According to the property of the vector field, we obtain that $0<x_{j+1}<x_{i+1}<\delta(Q-\alpha h)(k+h) /((m u-r D \delta) h-r D \delta k)$; that is,

$$
\begin{aligned}
& 0<x_{j+1}<x_{i+1}<\frac{\delta(Q-\alpha h)(k+h)}{(m u-r D \delta) h-r D \delta k}, \\
& \quad \text { for } 0<x_{i}<x_{j}<\frac{\delta(Q-\alpha h)(k+h)}{(m u-r D \delta) h-r D \delta k}, \tau>\bar{x}_{2} .
\end{aligned}
$$

For $x_{0} \in(0, \delta(Q-\alpha h)(k+h) /((m u-r D \delta) h-r D \delta k))$ and $\tau>\bar{x}_{2}$, we consider the Poincaré map $x_{i}=F\left(\tau, x_{i-1}\right), i=$ $1,2, \ldots, n$. If $x_{1}=x_{0}$, then system (2) has an order-1 periodic solution. If $x_{0} \neq x_{1}, x_{0}=x_{2}$, we obtain that system (2) has an order-2 periodic solution.

For convenience, we investigate the more general thing in the following, that is, $x_{0} \neq x_{1} \neq x_{2} \neq \cdots \neq x_{n}(n \geq 3)$.

(a) For $x_{0}<x_{1}$, it follows from $x_{2}<x_{1}$, which leads to the relation of $x_{0}, x_{1}, x_{2}$ to be one of the following cases according to the qualitative property of system (2). 


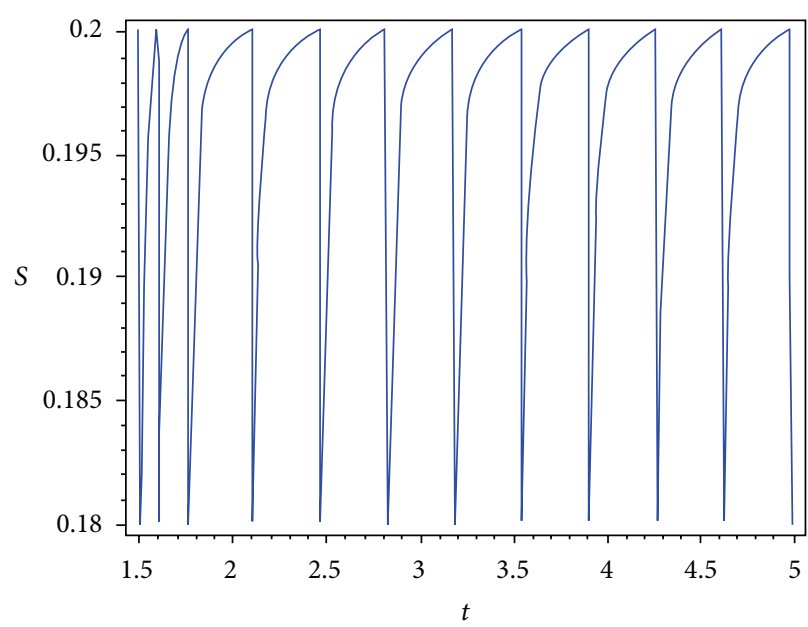

(a)

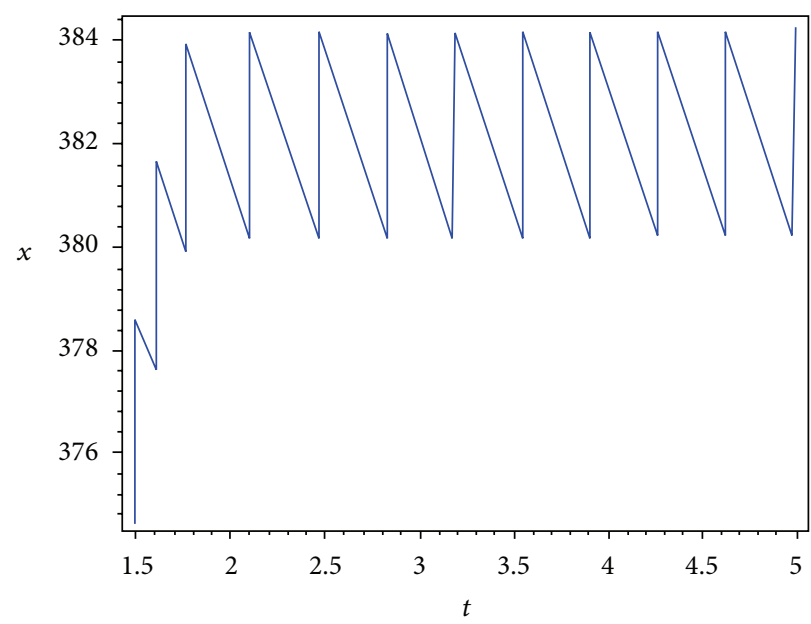

(b)

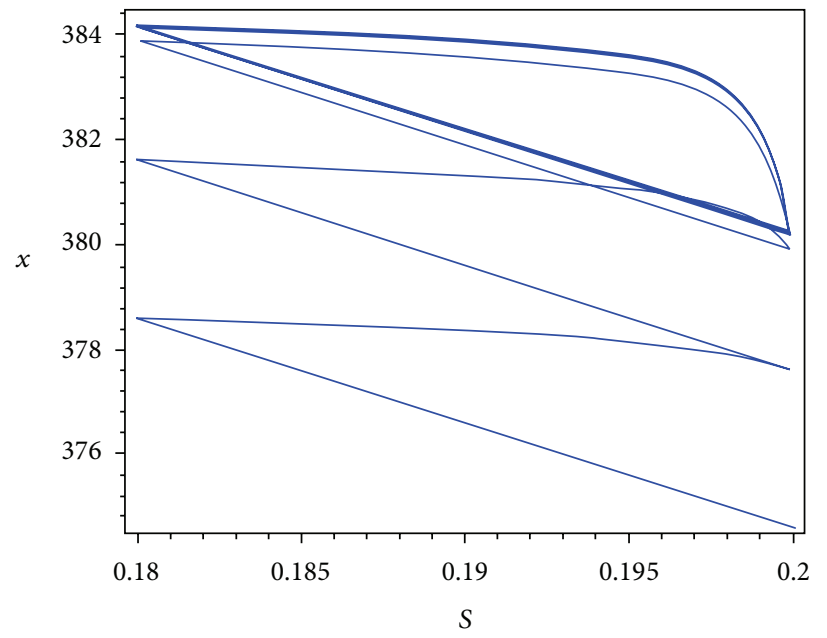

(c)

FigURE 2: For $h<k D /(\mu-D)$, the existence of order-1 periodic solution with the parameters $\mu=0.4, Q=6, k=4, r=0.01, D=0.03$, $E=0.1, \tau=4, h=0.2, \alpha=0.2$, and $\delta=0.01$.

(1 $\left.1_{\mathrm{a}}\right) x_{2}<x_{0}<x_{1}$, in the case, it follows from (15) that $x_{2}<x_{1}<x_{3}$. Repeating the above process, the following circumstances are obtained:

$$
\begin{aligned}
0 & <\cdots<x_{2 n}<\cdots<x_{2}<x_{0}<Z_{1}<\cdots<x_{2 n+1} \\
& <\cdots<\frac{\delta(Q-\alpha h)(k+h)}{(m u-r D \delta) h-r D \delta k} .
\end{aligned}
$$

$\left(2_{\mathrm{a}}\right)$ When $x_{0}<x_{2}<x_{1}$, similarly, we have

$$
\begin{aligned}
x_{0} & <x_{2}<\cdots<x_{2 n}<\cdots<x_{2 n+1}<\cdots<x_{3}<x_{1} \\
& <\frac{\delta(Q-\alpha h)(k+h)}{(m u-r D \delta) h-r D \delta k} .
\end{aligned}
$$

(b) When $x_{0}>x_{1}$, again from (15), we have $x_{1}<x_{2}$. The result leads to the relation of $x_{0}, x_{1}$, and $x_{2}$ to be one of the following cases:

$\left(1_{\mathrm{b}}\right)$

$$
\begin{aligned}
0< & <x_{2 n+1}<\cdots<x_{1}<x_{0}<x_{2}<\cdots<x_{2 n}<\cdots \\
< & \frac{\delta(Q-\alpha h)(k+h)}{(m u-r D \delta) h-r D \delta k} . \\
& \quad\left(2_{\mathrm{b}}\right) \text { If } x_{1}<x_{2}<x_{0}, \text { similarly, we also have } \\
0< & x_{1}<\cdots<x_{2 n+1}<\cdots<x_{2 n}<\cdots<x_{2}<x_{0} \\
& <\frac{\delta(Q-\alpha h)(k+h)}{(m u-r D \delta) h-r D \delta k} .
\end{aligned}
$$

Furthermore, in case $\left(1_{\mathrm{a}}\right)$ of (a), we obtain $\lim _{n \rightarrow \infty} x_{2 n}=\xi_{1}$ and $\lim _{n \rightarrow \infty} x_{2 n+1}=\xi_{2}$, where $0<\xi_{1}<\xi_{2}<\delta(Q-\alpha h)(k+$ $h) /((m u-r D \delta) h-r D \delta k)$. Hence we obtain $\xi_{1}=F\left(\tau, \xi_{2}\right)$ and 


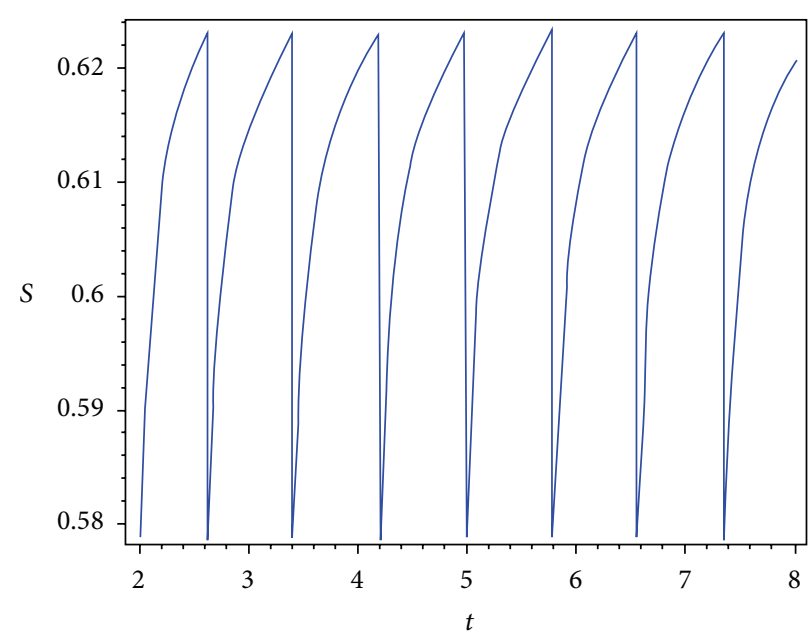

(a)

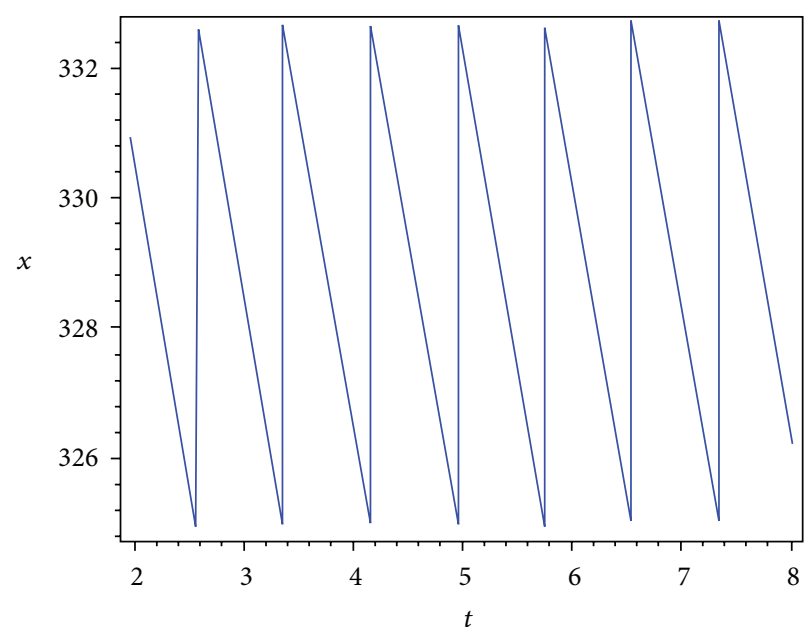

(b)

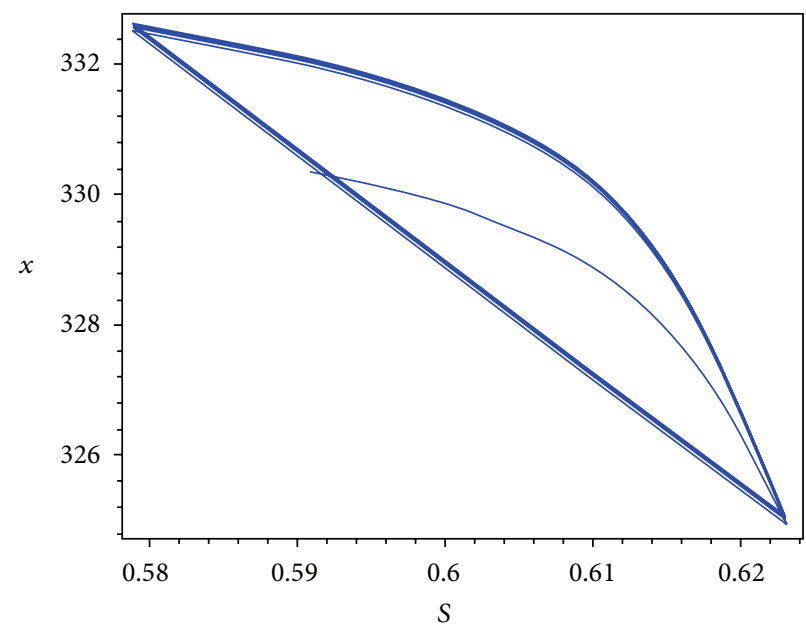

(c)

Figure 3: For $h \geq k D /(\mu-D)$, the existence of order-1 periodic solution with the parameters $\mu=0.4, Q=5, r=0.02, D=0.03, E=0.071$, $\tau=7.6, \alpha=0.2, \delta=0.02$, and $h=0.6233$.

$\xi_{2}=F\left(\tau, \xi_{1}\right)$; therefore, system (2) has an order-2 periodic solution, which is orbitally asymptotically stable. Similarly, for case $\left(2_{\mathrm{a}}\right)$ of $(\mathrm{a})$ and $\left(2_{\mathrm{b}}\right)$ of $(\mathrm{b})$, system (2) has an orbitally asymptotically stable order- 2 periodic solution. In case $\left(1_{b}\right)$ of (b), system (2) also has an order-2 periodic solution, which is orbitally asymptotically stable.

\section{Numerical Simulations and Discussion}

In this paper, we have formulated the mathematical model of microbial degradation with the impulsive state feedback control and obtained the existence of order-1 periodic solution and order- 2 periodic solution by using the geometry theory of semicontinuous dynamic system. System (2) without the impulsive effect has two equilibria, $E_{0}(Q / \alpha, 0)$ and $E^{*}\left(S^{*}, x^{*}\right)$; we show the microbe-free equilibrium $E_{0}(Q / \alpha, 0)$ is asymptotically stable if $R_{1}<1$. The positive equilibrium $E^{*}\left(S^{*}, x^{*}\right)$ is globally asymptotically stable if $R_{1}>1$, which means that pollutant concentration will reach unacceptable level if no measurement is adopted. In order to reduce the pollutant concentration to the predetermined threshold, which is less harmful to human health, the model of the microbial degradation is proposed. By using the geometric theory of semicontinuous dynamical system, we obtain system (2) exists an order-1 periodic solution for $h<k D /(\mu-D)$, which implies the control measurement is effective even if the initial concentration of pollutant is very high and the example is given as follows:

$$
\begin{aligned}
& \frac{d S}{d t}=6-\alpha S-\frac{4 S x}{0.01(4+S)}+0.0003 x \\
& \frac{d x}{d t}=\frac{4 S x}{(4+S)}-0.03 x
\end{aligned}
$$




$$
\begin{aligned}
& \Delta S=-0.1 S, \\
& \Delta x=4,
\end{aligned}
$$$$
S=0.2
$$

System (20) can be simulated in Figure 2. We see the trajectory starting through the initial value $(0.6,0.3)$ is attracted by the order-1 periodic solution (see Figure 2). When $h \geq$ $k D /(\mu-D)$, system (2) exists an order-2 periodic solution starting from the initial value $(0.13,0.36)$ with the parameters $\mu=0.4, Q=5, r=0.02, D=0.03, E=0.071, \tau=7.6$, $\alpha=0.2, \delta=0.02$, and $h=0.6233$ (see Figure 3).

\section{Conflict of Interests}

The authors declare that there is no conflict of interests regarding the publication of this paper.

\section{Acknowledgments}

This work is supported by the National Natural Science Foundation of China (no. 11371164), NSFC-Talent Training Fund of Henan (U1304104), Innovative talents of science and technology plan in Henan province (15HASTIT014), and the young backbone teachers of Henan (no. 2013GGJS-214).

\section{References}

[1] N. S. Strigul and L. V. Kravchenko, "Mathematical modeling of PGPR inoculation into the rhizosphere," Environmental Modelling and Software, vol. 21, no. 8, pp. 1158-1171, 2006.

[2] S. M. Kharrazi, H. Younesi, and J. Abedini-Torghabeh, "Microbial biodegradation of waste materials for nutrients enrichment and heavy metals removal. An integrated compostingvermicomposting process," International Biodeterioration and Biodegradation, vol. 92, pp. 41-48, 2014.

[3] J. Peng, G. Wu, and K. Chen, Studies on identification of a wetland plant rhizosphere microorgan-ism LF2, optimization of the fermental conditions and application effect in constructed wetland [M.S. thesis], Central China Normal University, Wuhan, China, 2011.

[4] Z. W. Wang, Q. Lin, and Y. X. Chen, Effect of organic-heavy metal combined polluted on rhizosphere microbial ecology [M.S. thesis], Zhejiang University, Zhejiang, China, 2005.

[5] K. Lika and I. A. Papadakis, "Modeling the biodegradation of phenolic compounds by microalgae," Journal of Sea Research, vol. 62, no. 2-3, pp. 135-146, 2009.

[6] X.-W. Huang, Q.-Y. Wei, K. H. Urata, Y. Tomoshige, X.-H. Zhang, and Y. Kawagoshi, "Kinetic study on nitrogen removal performance in marine anammox bacterial culture," Journal of Bioscience and Bioengineering, vol. 117, no. 3, pp. 285-291, 2014.

[7] S. Ponsá, B. Puyuelo, T. Gea, and A. Sánchez, "Modelling the aerobic degradation of organic wastes based on slowly and rapidly degradable fractions," Waste Management, vol. 31, no. 7, pp. 1472-1479, 2011.

[8] M. Seto and I. C. Handoh, "Mathematical explanation for the non-linear hydrophobicity-dependent bioconcentration processes of persistent organic pollutants in phytoplankton," Chemosphere, vol. 77, no. 5, pp. 679-686, 2009.
[9] M. Serhani, J. L. Gouze, and N. Raissi, "Dynamical study and robustness for a nonlinear wastewater treatment model," Nonlinear Analysis. Real World Applications, vol. 12, no. 1, pp. 487-500, 2011.

[10] J. B. Shukla, A. K. Misra, and P. Chandra, "Mathematical modeling and analysis of the depletion of dissolved oxygen in eutrophied water bodies affected by organic pollutants," Nonlinear Analysis: Real World Applications, vol. 9, no. 5, pp. 1851-1865, 2008.

[11] A. Goyal, R. Sanghi, A. K. Misra, and J. B. Shukla, "Modeling and analysis of the removal of an organic pollutant from a water body using fungi," Applied Mathematical Modelling, vol. 38, no. 19-20, pp. 4863-4871, 2014.

[12] A. K. Misra, P. Chandra, and J. B. Shukla, "Mathematical modeling and analysis of the depletion of dissolved oxygen in water bodies," Nonlinear Analysis. Real World Applications, vol. 7, no. 5, pp. 980-996, 2006.

[13] P. M. Beckett, W. Armstrong, and J. Armstrong, "Mathematical modelling of methane transport by phragmites: the potential for diffusion within the roots and rhizosphere," Aquatic Botany, vol. 69, no. 2-4, pp. 293-312, 2001.

[14] K. Bunwong, W. Sae-jie, and Y. Lenbury, "Modelling nitrogen dynamics of a constructed wetland: nutrient removal process with variable yield," Nonlinear Analysis: Theory, Methods \& Applications, vol. 71, no. 12, pp. e1538-e1546, 2009.

[15] E. M. Scott, E. A. S. Rattray, J. I. Prosser et al., "A mathematical model for dispersal of bacterial inoculants colonizing the wheat rhizosphere," Soil Biology and Biochemistry, vol. 27, no. 10, pp. 1307-1318, 1995.

[16] H. G. Guo and L. S. Chen, "Periodic solution of a chemostat model with Monod growth rate and impulsive state feedback control," Journal of Theoretical Biology, vol. 260, no. 4, pp. 502509, 2009.

[17] Z. Zhao, T. Y. Wang, and L. S. Chen, "Dynamic analysis of a turbidostat model with the feedback control," Communications in Nonlinear Science and Numerical Simulation, vol. 15, no. 4, pp. 1028-1035, 2010.

[18] K. B. Sun, A. Kasperski, Y. Tian, and L. S. Chen, "Modelling of the Corynebacterium glutamicum biosynthesis under aerobic fermentation conditions," Chemical Engineering Science, vol. 66, no. 18, pp. 4101-4110, 2011.

[19] K. Sun, Y. Tian, L. Chen, and A. Kasperski, "Nonlinear modelling of a synchronized chemostat with impulsive state feedback control," Mathematical and Computer Modelling, vol. 52, no. 1-2, pp. 227-240, 2010.

[20] R. Smith, Impulsive differential equations with application to self-cycling fermentation [Ph.D. thesis], Mcmaster University, Hamilton, Canada, 2001.

[21] K. Sung, J. Kim, C. L. Munster et al., "A simple approach to modeling microbial biomass in the rhizosphere," Ecological Modelling, vol. 190, no. 3-4, pp. 277-286, 2006.

[22] C. Wei and L. Chen, "Homoclinic bifurcation of prey-predator model with impulsive state feedback control," Applied Mathematics and Computation, vol. 237, pp. 282-292, 2014. 


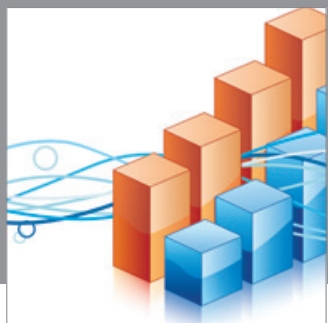

Advances in

Operations Research

mansans

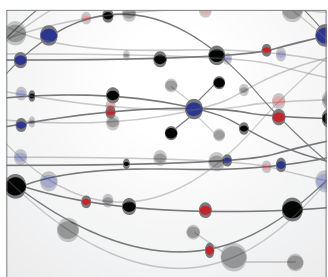

The Scientific World Journal
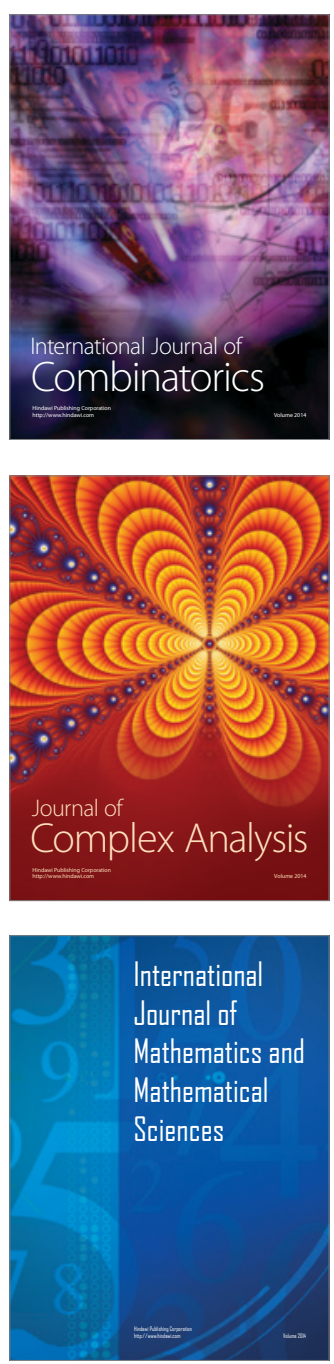
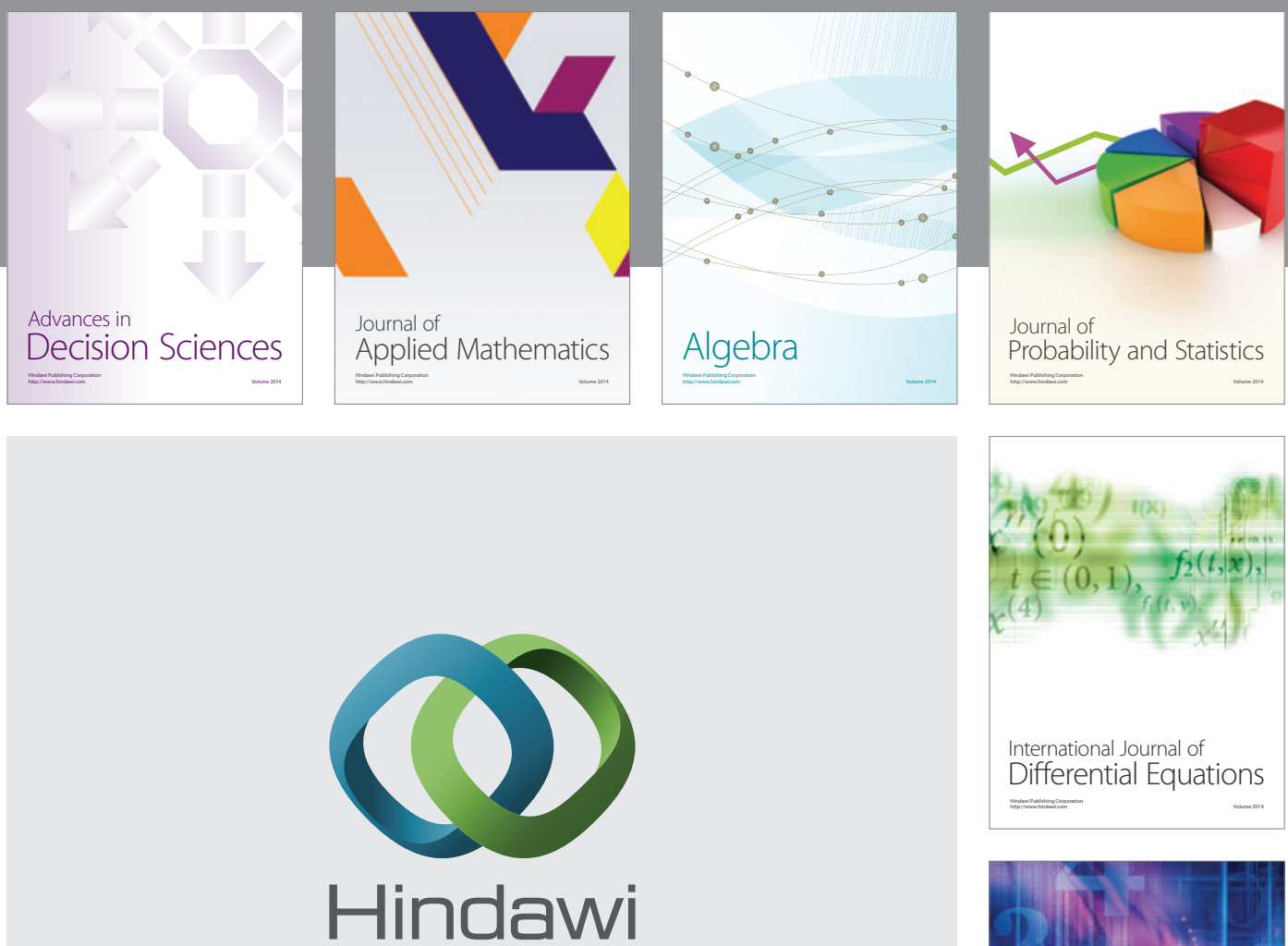

Submit your manuscripts at http://www.hindawi.com
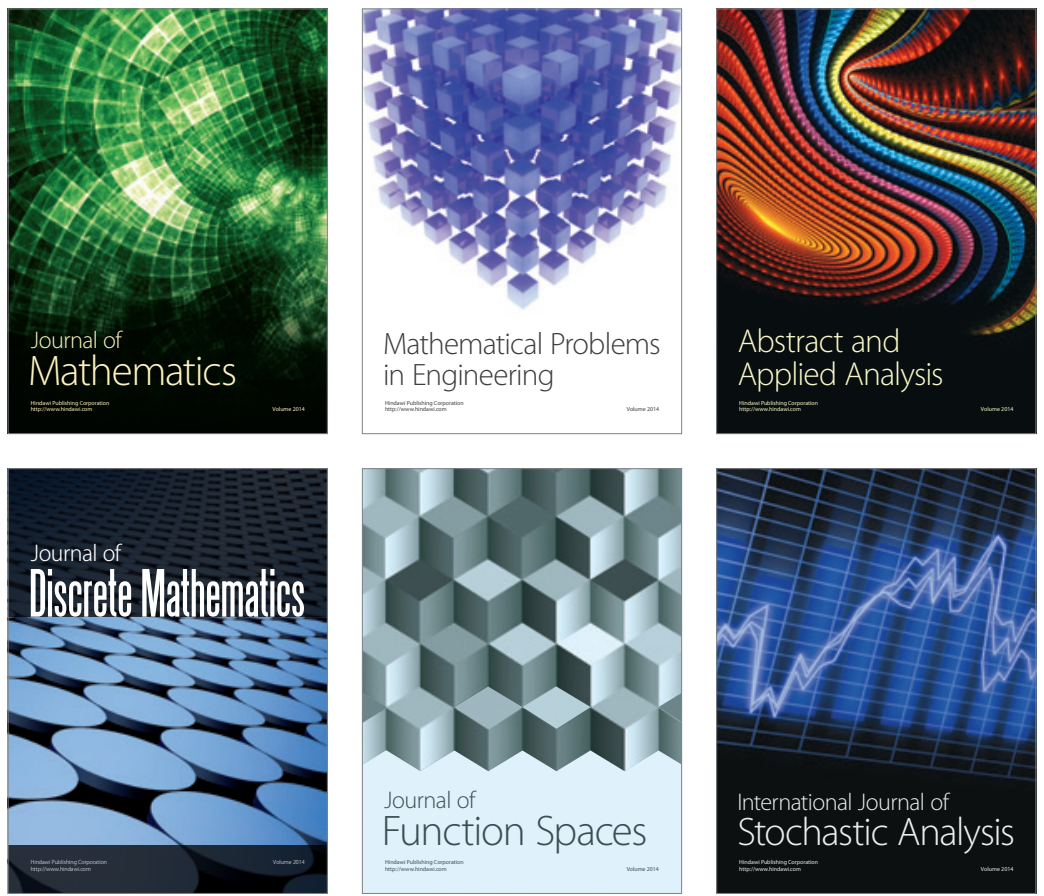

Journal of

Function Spaces

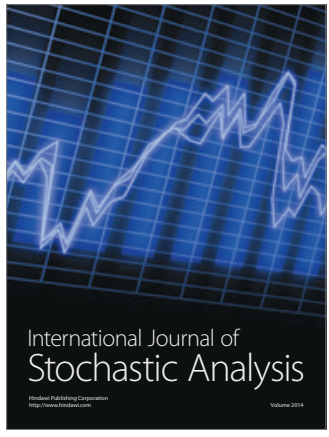

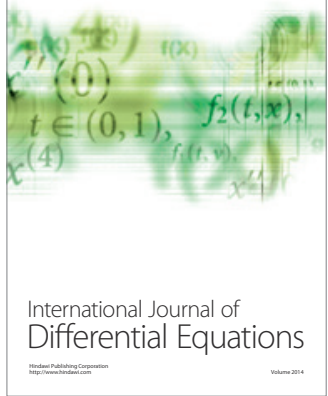
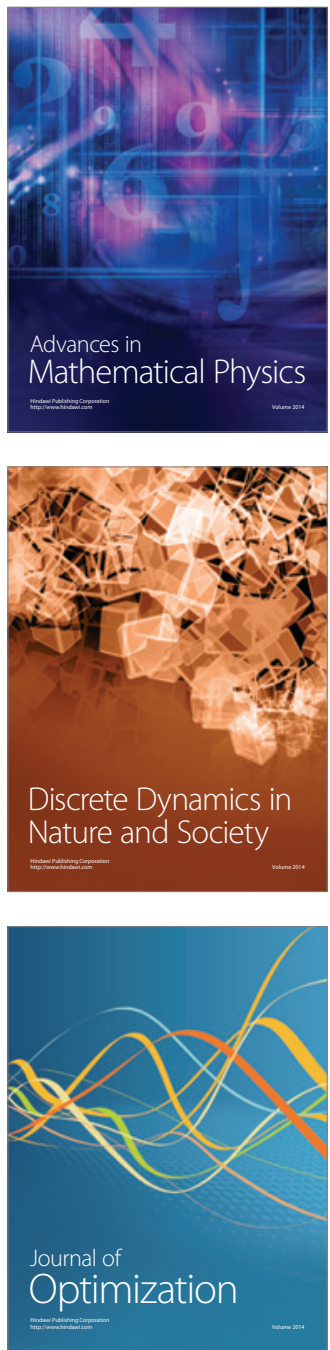